\title{
A New Approach for Finite Capacity Planning in MRP Environment
}

\author{
Hong-bum $\mathrm{Na}^{1}$, Hyoung-Gon Lee ${ }^{1}$, and Jinwoo Park ${ }^{1}$ \\ 1 Dept. of Industrial Engeering / Automation \& Systems Research Inst. \\ Seoul National University \\ San 56-1, SinRim-Dong, GwanAk-Gu, Seoul, 151-744, Korea \\ WWW home page: http://mailab.snu.ac.kr
}

\begin{abstract}
MRP (Material Requirement Planning) process generates a production plan which guarantees an exact quantity of right materials on needed time but it sometimes causes capacity problems in the shop floor because of its ignorance on capacity constraints. This paper presents a new planning method which involves the capacity planning during the MRP process. This planning method starts from generating resource-based data structure and grouping resources which can act as a bottleneck. Explosion of resources and adjustment of production schedules in accordance with the time horizon and the critical resources follow the former step.
\end{abstract}

\section{Introduction}

MRP (Material Requirement Planning) process is one of the key components in current manufacturing planning systems. The primary role of MRP is to generate a production plan so that we have the exact quantity of right materials at right place on time. The process of MRP is well defined and gives us exact timing information on purchasing or producing materials but it has some drawbacks. One of the most serious drawbacks would be its ignorance on capacity constraints. The MRP system performs part explosion process with fixed lead time on the assumption that resource capacity is infinite. Therefore the generated production plan may exceed the production capacity and the plan would become infeasible.

In order to solve the infinite capacity problems with regard to original MRP process, there have been many efforts for the past several decades mostly by industrial practitioners. Established studies aimed to integrate MRP and capacity planning in a closed loop planning system to make MRP more practical. In spite of these enormous efforts the problem has not been largely conquered yet, in the sense that we cannot get the solution in a reasonably short time to be of practical value. And the problem is still left to the hands of high-caliber consultants.

Please use the following format when citing this chapter:

Na, H.-b., Lee, H.-G. and Park, J., 2008, in IFIP International Federation for Information Processing, Volume 257, Lean Business Systems and Beyond, Tomasz Koch, ed.; (Boston: Springer), pp. 21-27. 
In a dynamic market environment, production orders tend to include high variety of product mixes and therefore production resources on shop floor level should be shared as much as possible to increase the utilization of resources. However, it can cause serious and complicated capacity problems also. This study aims to mitigate such problem by approaching the problem from a different perspective. Proposed approach starts by building a new resource view extracted from the basic MRP information (i.e. bills of material, routing) and then use scheduling algorithms by considering the capacities of resources and taking advantage of information obtained by this new data structure.

This paper is organized as follows. Section 2 presents a literature review about solution approaches to the finite capacity MRP problem. Section 3 introduces some basic steps which organize the finite capacity MRP process. In section 4, the framework of finite capacity MRP process is presented. Section 5 summarizes the work.

\section{Literature review}

Studies designed to solve finite capacity MRP can be mainly divided into two types: to solve the capacity-sensitive lot sizing problem mathematically and to adjust the production plans generated by infinite capacity MRP.

Capacity-sensitive lot sizing has been focused by extensive research. With capacity-sensitive lot sizing the capacity load is taken into account in the lot size computation. An early effort to model this problem was done by Billington et al. (1983) who formulated a capacity-constrained MRP system as a mixed-integer program (MIP). They used product structure compression techniques to reduce the size of the MIP and still maintain optimality. Tadrif et al. (1993) proposed a computationally fast procedure, which they labeled as MRP-C. They started with a capacity aggregated LP formulation, which was then solved via a greedy heuristic. The limitations to their model are: (1) it does not contain lot sizing constraints, (2) has the same lead time structure as the earlier models. Nagendra et al. (2001) also began with an LP formulation with different lead time model and lot sizing rules. They designed a concurrent procedure of finite capacity planning and lot sizing called MRP progressive capacity analyzer (PCA) and it outperformed other methods used in practice according to their reports. However, this result needs to be proved under dynamic production environments since these type of algorithms tend to show nervous behavior.

It is not easy to generalize the adjustment approaches since many diverse methods are possible to solve and prevent capacity problems, e.g. moving production orders between time buckets, changing routings, etc. An early proposal, schedulebased MRP (Hastings et al. 1982) used a form of forward loading to schedule the jobs on the available capacity. This guarantees feasible start and finish dates for the production orders. Sum and Hill (1993) describe a method that not only adjusts lot sizes to minimize set-ups but also determines the start and finish times of production orders while considering capacity constraints. Their algorithm iteratively splits or combines production orders to minimize set-up and inventory cost. Tall and 
Wortmann (1997) suggest that the capacity problems be resolved at the material planning stage itself through an integrated approach to MRP and finite capacity planning, but the performance of their model is not presented.

Another attempt basically distinctive from other research to solve finite capacity MRP is to suggest a unified database schema of BOM and routing (Tatsiopoulos, 1996). This paper examines the far-reaching consequences of unifying BOM and routings on the basic functions of production planning and control. Our proposed method may be viewed as one ramification of adjustment approaches.

\section{Basic steps}

In our approach, we divide the capacity adjustment process into three steps: 1) the structured resource formations step, 2) the resources grouping step, 3) schedule adjustment step. The structured resource step and the resource grouping step are preliminaries to involve the capacity constraints into the MRP and scheduled adjustment step is actual planning process to generate a production plan.

\subsection{The structured resource formations step}

The structured resource formations step is a basic step for capacity adjustment process by simplifying and integrating the production master data. In this step, the information contained in the BOM (Bills of Materials), routing and production orders are converted into a tree structured data before the part explosion process. The new data structure is called the Process oriented Inverted BOM (PI-BOM).

The PI-BOM is the main structure for implementing capacity planning. The PI$\mathrm{BOM}$ contains the information about processing time of certain resources to produce corresponding parts so that the requirements to meet the customer order quantity could be calculated into a resource usage. So total orders which should be planned in the planning period converts into the total amount of using resources in this step.
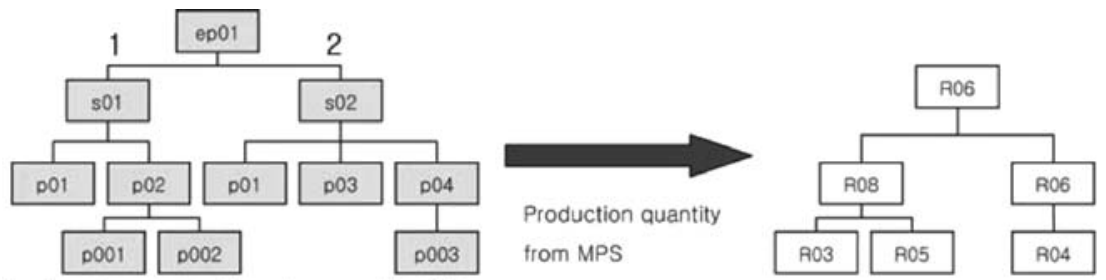

Routing of producing the sub-assembly $\mathrm{s} 01$ : R03(0.5). R03(0.6). R05(0.2). R08(0.7)

Routing of producing the sub-assembly s02 : R04(0.7), R06(0.3), R06(0.8)

Fig. 1. Converting production master data into the PI-BOM 
Figure. 1 shows an example of the conversion from a standard type of production master data into the PI-BOM. The PI-BOM contains only the key information needed for shop floor scheduling and has much simpler structure than ordinary engineering or manufacturing BOM.

\subsection{The resources grouping step}

Some resources have a problem of capacity overload because the production resources are not evenly used in the production shop floor. These resources trigger the capacity problem which affects the whole manufacturing process. This capacity overflow leads to a failure for meeting the customer order while keeping the due date. Especially the high-mix production environment requires high level of the resource sharing and therefore the capacity overload can occur on many resources. Resources which frequently face the capacity overload problem are considered to be critical resources of whole production process.

In the resources grouping step, we gather the resources into a resource group that can act as a number of critical resources by searching for interconnections and usage frequency of the resources from historical data or analyzed result of the PI-BOM. The critical resources are the resources that may cause bottlenecks in the production sequence and bring about capacity problems. Thus, critical resources are the main target to be involved for schedule adjustment. The critical resources and PI-BOM which is mentioned on former section are the basic tools to cope with the problems of overloaded production capacities.

\subsection{Schedule adjustment step}

In schedule adjustment step, we trace and use the information contained in the PI-BOM. We rearrange the exploded resource requirements in accordance with the time horizon and the critical resources, and then adjust the excess capacities in the planning period. First, adjusting procedure starts with the backward scheduling, the most simple inventory reduction process. Then further adjustments are made by moving orders which contain overloaded critical resource groups. We adjust resources levels and generate new material requirement plans considering production orders, capacity constraints, and pegging information. The pegging records contain part numbers, the types of resources and most importantly, the sources of all gross requirements. Using the pegging information, we could adjust the schedule for lower level PI-BOM items, which in turn let us adjust schedules for upper-level items, and finally to the customer orders.

\section{Framework of finite capacity MRP}

The proposed finite capacity MRP process starts from generating PI-BOM and ends with creating the plan of material requirements. Figure.2. shows the framework of finite capacity MRP process. 


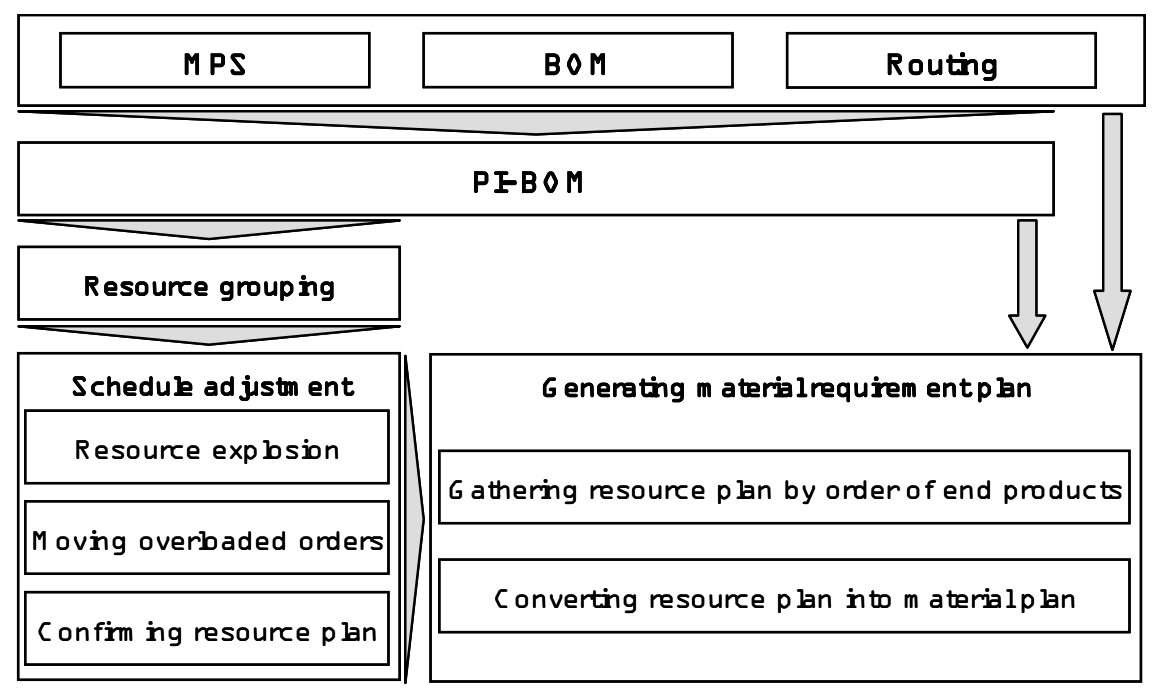

Fig. 2. The framework of finite capacity MRP

\subsection{Generating the PI-BOM}

To generate the PI-BOM, the information of Material BOM and Routing is essential. We can abstract the sub-assemblies and parts which build final items from the Material BOM. From the Routing information, we can determine the resource name and the processing time that are needed to produce sub-assemblies and parts. Therefore the records of PI-BOM contain the customer order (pegging information), parent resource, child resource, processing time and the level of process as main attributes. Conducting the part explosion and resource allocation at the same time, this data structure yields a feasible production plan by checking the capacity availability and adjusting the lead time when capacity overflow occurs.

The main attributes to build a finite capacity production plan are as follows:

- customer order(pegging information) : Information regarding the ownership of the order needs to be traced for the reason that the requirement plan from the same order has to be treated by group when capacity overflow occurs and scheduling adjustment follows.

- parent resource : Instead of parent item defined in Material BOM, it defines resource corresponding to the later process producing an item. This attribute is needed to examine the precedence of utilizing resources.

- child resource : Contrary to the parent resource concept, this object prescribes resource applied in the process that precedes each resource.

- processing time : It indicates the processing time that has to be processed by the given resource set. The sum of processing time determines the quantity used in specific resource for each period.

- level : It checks the locations which are occupied by current resources among the whole process for the production of end items. Also, especially, it can 
clarify the specific part to be processed by corresponding resource when same resource is assigned to the different processes.

\subsection{Creating the critical resource group}

Occupied capacity versus total capacity would be a reasonable criterion to select the critical resource group which is obtained from the historical data. Furthermore, the resource group is also utilized as a ground for which order will be chosen to be shifted. Namely, it acts as a priority criterion for altering schedule of orders when capacity overload happens.

\subsection{Generating the production plan}

Deriving a schedule of the resources is performed by same procedure of part explosion process which is used to make a material requirements plan, namely it can be also termed as resource explosion process. In the part explosion process, there are 4 types of records; Gross requirements, Scheduled receipts, Projected available balance and Planned order releases. These attributes should be converted into a resource-related terms for the resource explosion procedure. The types of records for resource explosion process are as follows.

- Capacity requirements from Gross requirements - resource usage time according to the actual order quantity

- Scheduled capacity from Scheduled receipts - utilizing reserved resource in advance (previously summed for capacity computation)

- Projected allowance from Projected available balance - anticipated by available capacity for the end of the given period

- Planned usage from Planned order release - final processing time corresponding to the actual order

Resource explosion proceeds iteratively until it produces total usage time for every lower level resource analogous to part explosion process.

The first step of adjusting schedule is a backward scheduling figuring out the resource on which the capacity overload occurs after the explosion process is conducted. For the reason that a single occurrence of schedule adjustment leads to alteration of every production schedule involving individual orders, all the orders involved in the resource with capacity overload should be traced. Furthermore, every production schedule prior to the resource tackled by capacity overload has to be moved forward.

Finally, the result of resource explosion considering the schedule adjustment is converted into requirement plan of material. This period-to-period conversion is done by deciding appropriate resource, referring to customer order and PI-BOM, is to be used for producing proper part. As a result, the same shape of time-phased requirement plan of production order is computed as the standard MRP process. 


\section{Conclusion}

In this study, we presented more convenient planning method which considers the capacity problem. To involve the capacity issues into the MRP process, three basic steps were suggested : 1) The structured resource formations step, 2) The resources grouping step, 3) Schedule adjustment step. Records of standard MRP process were converted into a PI-BOM in the structured resource formations step. The records of the PI-BOM consist of the usage of resources and pegging information. In the resources grouping step, resources that occurs capacity problem were grouped into critical resource groups. Finally, the resources were exploded and adjusted to generate a plan of material requirements which satisfies the resource constraints.

Finite capacity MRP based on PI-BOM can improve the performance of manufacturing planning and control system based on standard MRP system. Computational burden for scheduling algorithms may be reduced by taking into consideration of the upper level requirements of production orders up to final assembly schedules of the final products. Work is continuing with implement and further refinement of procedures. Experimental results will be compared with the current MRP and capacity planning methods.

\section{References}

1. Billington, P. J., McClain, J. O. and Thomas, L. J., 1983, Mathematical programming approaches to capacity-constrained MRP systems. Management Science, 29, 1126-1141.

2. Hastings, Nicholas A.J., Marshall, Peter and Willis, Robert J., 1982, Schedule Based M.R.P. : An integrated Approach to Production Scheduling and Material Requirements Planning. Journal of The Operational Research Society, 33, 10211029.

3. Nagendra, P.B. and DAS, S. K., 2001, Finite capacity scheduling method for MRP with lot size restrictions. International Journal of Production Research, 39, 16031623.

4. Sum, C. C. and Hill, A. V., 1993, A new framework for manufacturing planning and control systems. Decision Sciences, 24, 739-760

5. Tatsiopoulos, I. P., 1996, On the unification of bills of materials and routings. Computers in Industry, 31, 293-304.

6. Tall, Martin and Wortmann, Johan C., 1997, Integrating MRP and finite capacity planning. Production Planning \& Control, 8, 245-254.

7. Tardif, V., Spearman, M., Coullard, C. and Hopp, W. A., 1993, Framework for capacitated MRP. Working Paper, Northwestern University. 